Check for updates

The BMJ

Cite this as: $B M J 2020 ; 371: m 4470$ http://dx.doi.org/10.1136/bmj.m4470 Published: 17 November 2020

\title{
Long covid: Damage to multiple organs presents in young, low risk patients
}

\section{Gareth lacobucci}

Young, low risk patients with ongoing symptoms of covid-19 had signs of damage to multiple organs four months after initially being infected, a preprint study has suggested. ${ }^{1}$

Initial data from 201 patients suggest that almost $70 \%$ had impairments in one or more organs four months after their initial symptoms of SARS-CoV-2 infection.

The results emerged as the NHS announced plans to establish a network of more than 40 long covid specialist clinics across England this month to help patients with long term symptoms of infection.

The prospective Coverscan study examined the impact of long covid (persistent symptoms three months post infection) across multiple organs in low risk people who are relatively young and had no major underlying health problems. Assessment was done using results from magnetic resonance image scans, blood tests, and online questionnaires.

The research has not yet been peer reviewed and could not establish a causal link between organ impairment and infection. But the authors said the results had "implications not only for [the] burden of long covid but also public health approaches which have assumed low risk in young people with no comorbidities."

The study enrolled participants at two UK sites in Oxford and London between April and August 2020. Two hundred and one individuals (mean age 44 (standard deviation 11.0) years) completed assessments after SARS-CoV-2 infection a median of 140 days after initial symptoms.

Participants were eligible if they tested positive for SARS-CoV-2 by random polymerase chain reaction swab ( $n=62)$, a positive antibody test $(n=63)$, or had typical symptoms and were determined to have covid-19 by two independent clinicians $(n=73)$.

The prevalence of pre-existing conditions was low (obesity: $20 \%$, hypertension: $6 \%$, diabetes: $2 \%$, heart disease: $4 \%$ ), and less than a fifth (18\%) of individuals had been hospitalised with covid-19.

The most commonly reported ongoing symptoms-regardless of hospitalisation status-were fatigue (98\%), muscle ache ( $88 \%$ ), shortness of breath $(87 \%)$, and headache $(83 \%)$. There was evidence of mild organ impairment in the heart (32\% of patients), lungs (33\%), kidneys (12\%), liver (10\%), pancreas $(17 \%)$, and spleen $(6 \%)$.

Two thirds (66\%) of participants had impairment in one or more organ systems and there was evidence of multiorgan impairment in a quarter $(25 \%)$ of individuals, with varying degrees of overlap across multiple organs.
Multiorgan impairment was significantly associated with risk of prior covid-19 hospitalisation $(\mathrm{P}<0.05)$. Measures of inflammation in the kidneys and pancreas, and ectopic fat in the pancreas and liver, were also higher in hospitalised individuals $(\mathrm{P}<0.05)$.

The results "signal the need for monitoring and follow-up in at least the medium and longer term, especially for extrapulmonary sequelae" and demonstrate that long covid requires management across clinical specialties, the authors said.

The authors observed that neither symptoms nor blood investigations were predictive of organ impairment.

"Causality of the relationship between organ impairment and infection cannot be deduced, but may be addressed by longitudinal follow-up of individuals with organ impairment," the authors said.

Other limitations of the study included that diagnosis of covid-19 was by multiple methods, partly due to limited access to laboratory testing during the pandemic.

The research was funded by the UK's National Consortium of Intelligent Medical Imaging through the Industry Strategy Challenge Fund, Innovate UK, and the European Union's Horizon 2020 research and innovation programme.

Dennis A, Wamil M, Kapur S, Alberts J, Badley AD. Multi-organ impairment in low-risk individuals with long COVID. https://www.medrxiv.org/content/10.1101/2020.10.14.20212555v1.full.pdf.

This article is made freely available for use in accordance with BMJ's website terms and conditions for the duration of the covid-19 pandemic or until otherwise determined by BMJ. You may use, download and print the article for any lawful, non-commercial purpose (including text and data mining) provided that all copyright notices and trade marks are retained. 